\title{
Acute disseminated encephalomyelitis with delayed onset and feasibility of the Miethke shunt and sensor reservoir system: a case report
}

\author{
Gunnar Liminga ${ }^{1}$ - Anna Grabowska ${ }^{2}$ - Dýrleif Pétursdóttir ${ }^{3} \cdot$ Kristina G. Cesarini $^{4}$ - Elham Rostami ${ }^{4,5}$. \\ Christoffer Ehrstedt ${ }^{1}$ (D)
}

Received: 2 February 2021 / Accepted: 25 April 2021 / Published online: 16 June 2021

(C) The Author(s) 2021

\begin{abstract}
Acute disseminated encephalomyelitis (ADEM) is an immune-mediated demyelinating central nervous system disorder with predilection for early childhood. Delayed onset of ADEM is rare, and herein we present a previously healthy 5-year-old boy, with an unusual clinical course of ADEM with high intracranial pressure (ICP) and acute visual loss that was at first diagnosed as idiopathic intracranial hypertension without papilledema (IIHWOP). The boy underwent acute neurosurgical intervention with ventriculoperitoneal (VP) shunt using Miethke valve and sensor reservoir system and received high-dose steroid treatment with symptom relieve within days. This is the first case report using this system in such a young child, and we find it feasible and valuable also in younger children when VP shunt with ICP measurement is indicated.
\end{abstract}

Keywords Acute demyelinated encephalomyelitis · Delayed onset · High intracranial pressure · Ventriculoperitoneal shunt . Sensor reservoir

\section{Introduction}

Acute disseminated encephalomyelitis (ADEM) is an immune-mediated demyelinating central nervous system disorder with predilection for early childhood. Common presenting symptoms are encephalopathy, fever, headache, nausea, vomiting, and ataxia. Diagnosis is made by a combination of

Elham Rostami and Christoffer Ehrstedt contributed equally to this work.

Christoffer Ehrstedt

christoffer.ehrstedt@kbh.uu.se

1 Department of Women's and Children's Health, Uppsala University , Uppsala, Sweden

2 Department of Surgical Sciences, Radiology, Uppsala University, Uppsala, Sweden

3 Department of Neuroscience, Ophthalmology, Uppsala University, Uppsala, Sweden

4 Department of Neuroscience, Neurosurgery, Uppsala University, Uppsala, Sweden

5 Department of Neuroscience, Karolinska Institute, Stockholm, Sweden clinical and magnetic resonance imaging (MRI) findings of the brain and medulla, and provided other etiologies of inflammatory and infectious causes have been excluded $[1,2]$.

Herein, we present a previously healthy 5 -year-old boy, with an unusual clinical course of ADEM, eventually causing a very high intracranial pressure (ICP) resulting in an acute neurosurgical intervention with ventriculoperitoneal (VP) shunt.

\section{Case presentation}

Two months prior to symptom debut, the boy had a flu-like episode. Medical advice was sought at the local county hospital after 3 weeks of increasing symptoms with headache and nausea. Routine blood sampling and neurologic and ophthalmologic examinations were normal. Magnetic resonance imaging revealed optic nerve sheath distension and a minor bilateral intraocular protrusion of the optic nerves (Fig. 1a-c). A lumbar puncture (LP) demonstrated an elevated opening pressure of $39 \mathrm{~cm} \mathrm{H}_{2} \mathrm{O}$ and slightly elevated white cell count (2 poly, 8 mono) in the cerebrospinal fluid (CSF). He was diagnosed with idiopathic intracranial hypertension without 

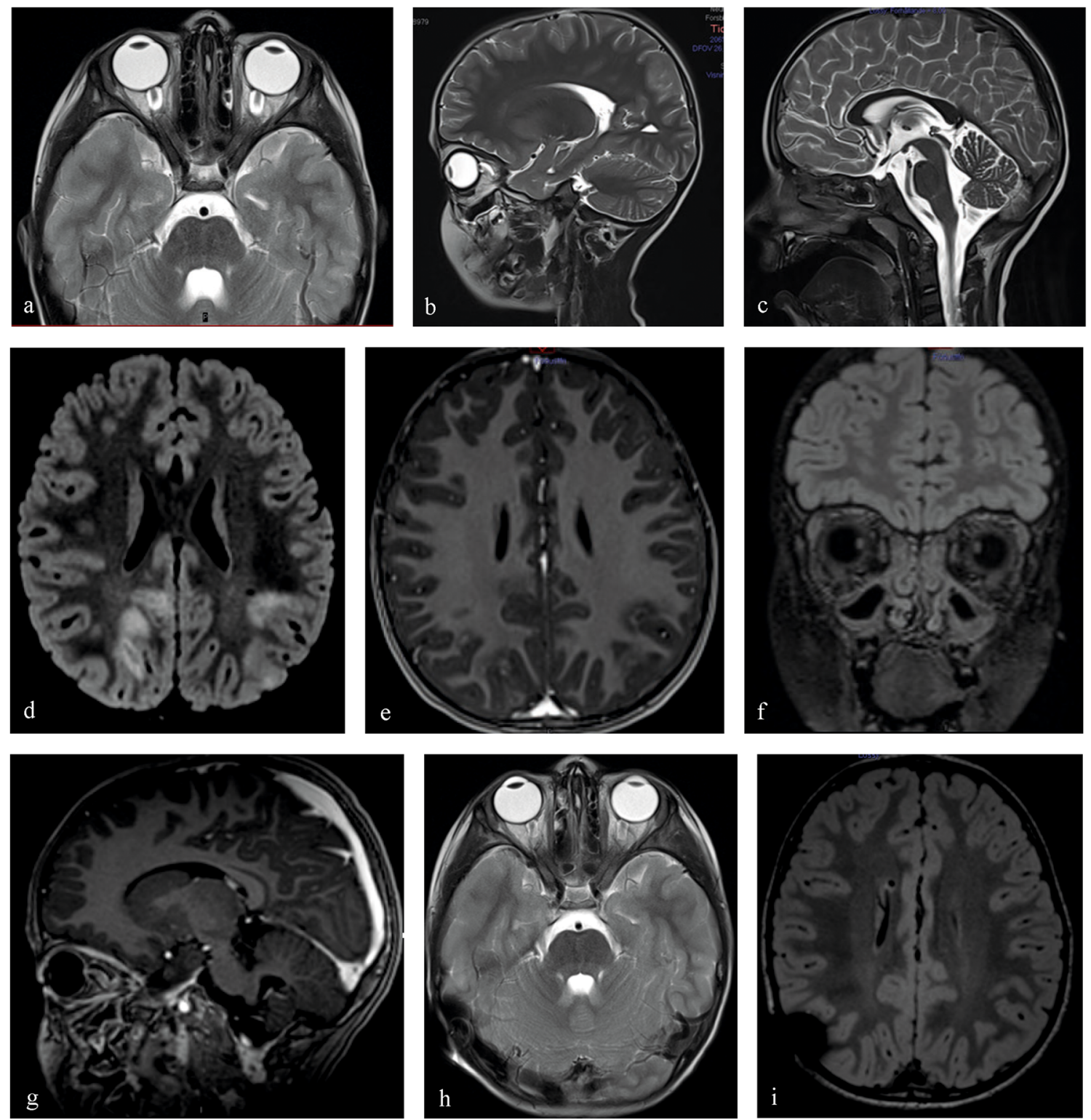

Fig. 1 a-c T2-weighted sequences from the initial MRI of the brain, performed during investigation of the headache and nausea, demonstrate findings consistent with IIH. a Transverse T2-weighted image shows significant distention of the perioptic nerve sheaths with flattening of the posterior sclera of both globes; $\mathbf{b}$ sagittal T2-weighted image showing same findings and in addition vertical tortuosity of the intraorbital optic nerve; c sagittal T2-weighted image with mild reduction in the height of the hypophysis. d-g MRI sequences obtained at the time of rapid deterioration with impairment of vision and encephalopathy. d Transverse FLAIR image demonstrates multiple bilateral subcortical hyperintense lesions dominating in the parietal and occipital lobes; e transverse T1

papilledema (IIHWOP), and treatment with acetazolamide (25 $\mathrm{mg} / \mathrm{kg} /$ day) was initiated.

One month after initiation of acetazolamide treatment, his medical condition deteriorated rapidly. He became encephalopathic within $24 \mathrm{~h}$ and experienced worsening of headache, nausea, and impaired vision. Ophthalmologic assessment revealed a visual acuity of 0.1 decimal in the right eye and only gadolinium $(\mathrm{Gd})$ image shows incomplete ring or nodular contrast enhancement in some of the subcortical lesions; $\mathbf{f}$ coronal FLAIR image shows bilateral optic nerve head protrusion into the globes; $\mathbf{g}$ sagittal T1 Gd image showing protrusion of the optic nerve head into the globes and diffuse contrast enhancement in both optic nerves. $\mathbf{h}-\mathbf{i}$ : Follow-up MRI 2 months after VP shunt placement and steroid treatment. $\mathbf{h}$ T2 transverse image shows regress of distention of perioptic nerve sheaths, posterior globe flattening, and optic nerve head protrusion into the globe; i FLAIR transverse image shows complete regression of the intracerebral signal changes

ability for light perception in the left eye, with a stage 5 papilledema bilaterally. Lumbar puncture demonstrated an opening pressure of $>50 \mathrm{~cm} \mathrm{H}_{2} \mathrm{O}$. An acute CT scan showed signs of high ICP. At this stage, he was transferred to Uppsala University Hospital, a tertiary referral center for children and adolescents in need of neurosurgical and pediatric neurology care and treatment. 
An acute MRI revealed extensive signs of neuroinflammation (Fig. 1d-g). A new diagnostic work-up was performed (Table 1), and a diagnosis of ADEM with bilateral optic neuritis $(\mathrm{ON})$ causing high ICP and visual loss was made. Due to the severe situation with an eminent threat of losing visual function, he received a VP shunt within hours from arrival to the pediatric intensive care unit. An ICP sensor reservoir/ prechamber system (Christoph Miethke, Potsdam, Germany) was connected to a Miethke, proGAV 2.0 valve, with opening pressure set at $18 \mathrm{~cm} \mathrm{H}_{2} \mathrm{O}$ and proSA, shunt assistant, setting of $10 \mathrm{~cm} \mathrm{H}_{2} \mathrm{O}$. The ICP sensor reservoir enabled daily ICP measurements postoperatively and demonstrated normal values (Fig. 2). Due to the MRI findings, a 5-day treatment with high-dose IV methylprednisolone $(30 \mathrm{mg} / \mathrm{kg} \times 1)$ was initiated, followed up with 2 weeks of high-dose prednisolone $(1 \mathrm{mg} / \mathrm{kg})$ with a tapering for an additional 6 weeks.

Table 1 A summary of the diagnostic work-up performed when the patient's medical condition deteriorated

\begin{tabular}{|c|c|c|}
\hline & & Normal range \\
\hline \multicolumn{3}{|l|}{ Neuroinflammation (CSF) } \\
\hline CXCL13 & 94 & $<7.8$ \\
\hline NMO-ab (aquaporin4-ab) & Negative & \\
\hline MOG-ab & Negative & \\
\hline Anti-neuronal ab & Negative & \\
\hline \multicolumn{3}{|l|}{$\mathrm{CSF}$} \\
\hline Glucose & Normal & \\
\hline Albumin & Normal & \\
\hline Lactate & Normal & \\
\hline White blood cells & 16 poly, 41 mono & $<6$ \\
\hline IgG index & 0.78 & $<0.63$ \\
\hline Oligoclonal bands & Negative & \\
\hline \multicolumn{3}{|l|}{ Infectious } \\
\hline Toxoplasmosis & Negative & \\
\hline Meningoencephalitis block* & Negative & \\
\hline TBE and Lyme & Negative & \\
\hline Parvo & Negative & \\
\hline Rubella & Negative & \\
\hline \multicolumn{3}{|l|}{ Other } \\
\hline $\mathrm{CBC}$ & Normal & \\
\hline Creatinine & Normal & \\
\hline ASAT, ALAT & Normal & \\
\hline TFT & Normal & \\
\hline ACE & Normal & \\
\hline
\end{tabular}

$N M O$ neuromyelitis optica, $M O G$ myelin oligodendrocyte glycoprotein, $T B E$ tick-borne encephalitis, $C B C$ complete blood count, $A S A T$ aspartate aminotransferase, $A L A T$ alanine aminotransferase, $T F T$ thyroid functions tests, $A C E$ angiotensin-converting enzyme

*Meningoencephalitis block; $H S V-1$ herpes simplex virus type 1, HSV-2, entero-, varicella zoster-, cytomegalo- and Epstein-Barr virus
There was a successive alleviation of symptoms. Visual acuity assessed 6 days postoperatively was 0.8 and 0.63 on the right and left eyes, respectively, with regression of papilledema to stage 4 on the right eye and less pronounced stage 5 on the left eye (Fig. 3).

Further clinical and neuroradiological follow-ups (Fig. 1hi) have been uneventful with the exception of a few episodes with recurrent headache. At 1-year follow-up, there was full recovery with normal visual function and without any neurologic deficits. Multiple ICP measurements with the Miethke sensor system were within normal range, both at episodes with recurrent headache and at 1-year follow-up (Fig. 2).

\section{Discussion}

Here we have presented an atypical case of ADEM with ON initially mimicking IIH/IIHWOP. Somewhat confusing was the initial prolonged clinical course together with normal neuroradiological findings. However, pleocytosis in CSF is not consistent with IIH/IIHWOP, and our case highlights the importance of diagnostic scrutiny in this regard. High levels of CXCL13 and white matter changes on MRI supported the final diagnosis of a demyelinating CNS disorder.

Severe presentations of ADEM resulting in admission to intensive care units have been described [3]; however, alleviation of very high ICP with neurosurgical interventions such as hemicraniectomy or VP shunt are limited to case reports [4, 5]. Timing of neurosurgical intervention may be a meticulous task when a patient presents with signs of increased ICP secondary to neuroinflammatory disease. It is possible that our patient would have responded well to just steroid treatment. However, due to the quick clinical deterioration and ophthalmological findings, the decision was made to relieve the high ICP with a VP shunt and treat the underlying neuroinflammation. One could also argue that an acute but temporary CSF diversion, such as ventriculostomy or lumbar drainage, could have been used. However, in the light of an already protracted clinical course and IIH being a possible differential diagnosis at the time of neurosurgical intervention, a VP shunt was considered the best alternative. Our case supports measuring lumbar puncture opening pressure as a clinical routine in children with ADEM, as suggested by Orbach et al. [6].

This is the first report describing the use of Miethke, proGAV 2.0, proSA, and ICP sensor reservoir/prechamber system in a preschool child. For a few years, Christoph Miethke $\mathrm{GmbH}$ has provided a pressure sensor system that allows ICP to be measured without performing lumbar punctures or invasive ICP monitoring in the neurointensive care unit. It also has the advantage of being MRI compatible. Thus, the Miethke shunt valve and sensor reservoir system are highly suitable for complex cases when repeated MRI examinations are expected and/or valve adjustments are predicted to be 
ICP

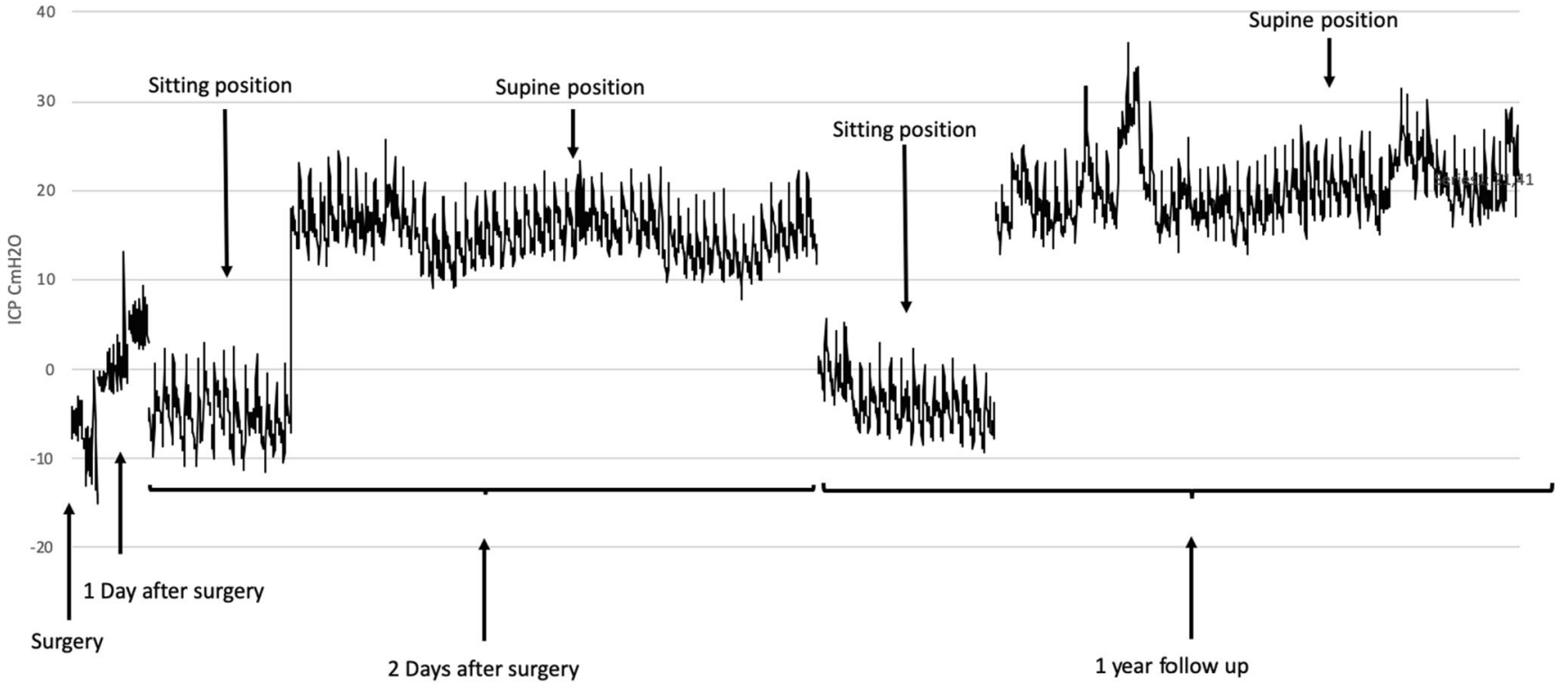

Fig. 2 Timeline of ICP measurements with the Miethke sensor system following surgery. ICP values of -10 up to $+5 \mathrm{~cm} \mathrm{H}_{2} \mathrm{O}$ was noted directly postoperatively. Daily ICP measurements postoperatively demonstrated values of -10 to $0 \mathrm{~cm} \mathrm{H}_{2} \mathrm{O}$ in sitting position and +10 to $20 \mathrm{~cm} \mathrm{H}_{2} \mathrm{O}$ in

supine position. Measurements were performed during changes in patient position in order to assess shunt valve function and suitable settings. Clinical symptoms and ICP measurements together guided the valve settings

challenging due to insufficient guidance of neuroimaging findings. The ICP measurement is not dependent on the position of the patient and can be performed in supine, sitting, and standing positions. This is even more beneficial in children when LP is performed for ICP measurement, and they need to be sedated. Furthermore, the ICP sensor represents the intracranial pressure better compared to lumbar measurements. Santes et al. reported successful use of this system in 25 patients including children, youngest 13 years of age, and that ICP-guided valve adjustments positively changed the clinical state in 18 of 25 patients [7].

\section{Conclusion}

Delayed onset of ADEM occasionally occurs. In patients presenting with signs of raised ICP and/or severe visual impairment, there is a clinical rationale for measuring the lumbar opening pressure. In cases when neurosurgical intervention is necessary, it is advisable to consider the possibility of a MR compatible Miethke shunt valve and sensor reservoir system to measure ICP in a non-invasive way, especially in younger children.

Fig. 3 Fundus photography of the patient 6 days after the VP shunt operation
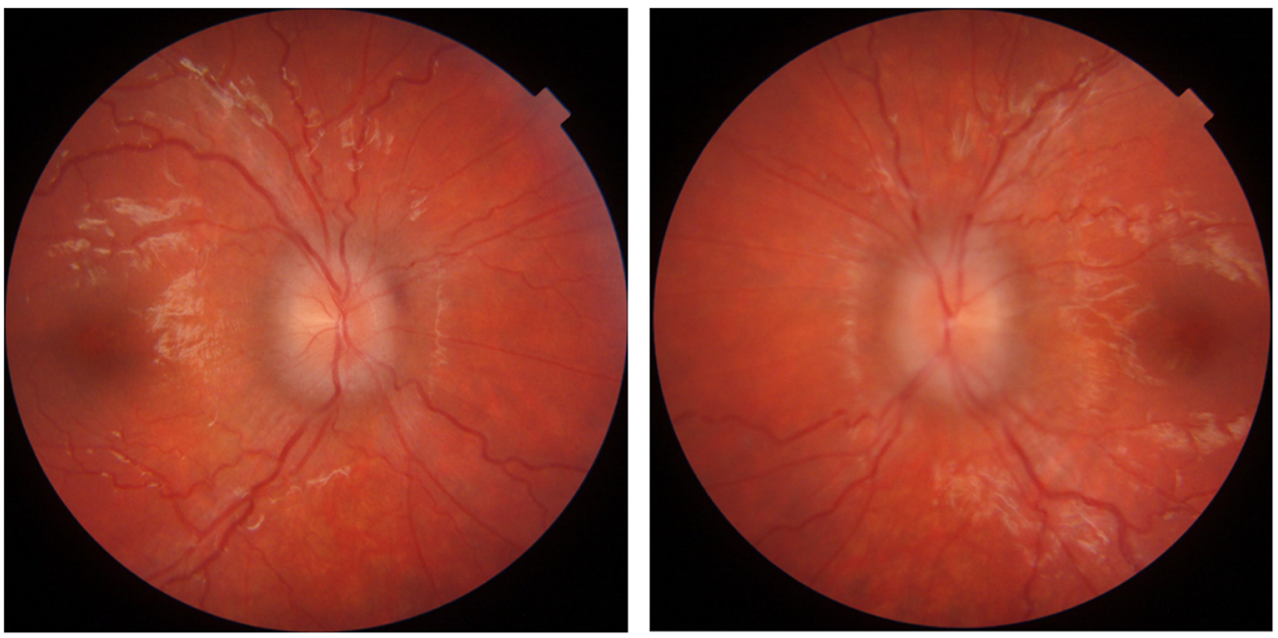
Acknowledgements We would like to thank senior lecturer Donald MacQueen for his English language editing.

Funding Open access funding provided by Uppsala University.

\section{Declarations}

Informed consent Informed consent was obtained from the parents to publish the information.

Conflict of interest On behalf of all authors, the corresponding author states that there is no conflict of interest.

Open Access This article is licensed under a Creative Commons Attribution 4.0 International License, which permits use, sharing, adaptation, distribution and reproduction in any medium or format, as long as you give appropriate credit to the original author(s) and the source, provide a link to the Creative Commons licence, and indicate if changes were made. The images or other third party material in this article are included in the article's Creative Commons licence, unless indicated otherwise in a credit line to the material. If material is not included in the article's Creative Commons licence and your intended use is not permitted by statutory regulation or exceeds the permitted use, you will need to obtain permission directly from the copyright holder. To view a copy of this licence, visit http://creativecommons.org/licenses/by/4.0/.

\section{References}

1. Krupp LB, Tardieu M, Amato MP, Banwell B, Chitnis T, Dale RC, Ghezzi A, Hintzen R, Kornberg A, Pohl D, Rostasy K, Tenembaum $\mathrm{S}$, Wassmer E, for the International Pediatric Multiple Sclerosis Study Group (2013) International Pediatric Multiple Sclerosis Study Group criteria for pediatric multiple sclerosis and immunemediated central nervous system demyelinating disorders: revisions to the 2007 definitions. Mult Scler 19(10):1261-1267

2. Cole J, Evans E, Mwangi M, Mar S (2019) Acute disseminated encephalomyelitis in children: an updated review based on current diagnostic criteria. Pediatr Neurol 100:26-34

3. Absoud M, Parslow RC, Wassmer E, Hemingway C, Duncan HP, Cummins C et al (2011) Severe acute disseminated encephalomyelitis: a paediatric intensive care population-based study. Multiple Scler (Houndmills, Basingstoke, England) 17(10):1258-1261

4. Dombrowski KE, Mehta AI, Turner DA, McDonagh DL (2011) Life-saving hemicraniectomy for fulminant acute disseminated encephalomyelitis. Br J Neurosurg 25(2):249-252

5. Sekula RF Jr, Marchan EM, Baghai P, Jannetta PJ, Quigley MR (2006) Central brain herniation secondary to fulminant acute disseminated encephalomyelitis: implications for neurosurgical management. Case report. J Neurosurg 105(3):472-474

6. Orbach R, Schneebaum Sender N, Lubetzky R, Fattal-Valevski A (2019) Increased intracranial pressure in acute disseminated encephalomyelitis. J Child Neurol 34(2):99-103

7. Antes S, Stadie A, Müller S, Linsler S, Breuskin D, Oertel J (2018) Intracranial pressure-guided shunt valve adjustments with the Miethke sensor reservoir. World Neurosurg 109:e642-ee50

Publisher's note Springer Nature remains neutral with regard to jurisdictional claims in published maps and institutional affiliations. 